\title{
Will melatonin change the current attitude towards menopausal hormone replacement therapy?
}

\begin{abstract}
Hormonal replacement therapy improves quality of life and prevents several serious diseases in women with premature or physiological menopause. At the same time, it is a factor increasing the risk of carcinogenesis, especially concerning breast cancer. The current attitude towards menopausal hormone therapy (MHT) is thus ambiguous, and the decision about its use needs to be carefully evaluated, considering the relevance of its potential risks and benefits in each particular clinical scenario. Several recent studies have pointed out the potential use of melatonin for both prevention and treatment of different types of cancer, the effects on breast cancer being the most documented ones. These data may soon become a basis for preventive treatment for different categories of women at risk. Those underoing MHT are epecially concerned. The availability of melatonin as a generally well-tolerated and nontoxic anticancer drug is thus likely to incline the balance of the current decision-making schemes concerning the adequacy of MHT in favor of its broader use, including the start at a relatively advanced age or resumption after a long break period. Beneficial effects of melatonin on other pathological processes related with aging will probably also come into play. Combined estrogen-melatonin-progestogen therapy (EMPT) may thus become the MHT of choice in the near future.
\end{abstract}

Volume 9 Issue $6-2018$

\author{
Jan Tesarik,' Raquel Mendoza-Tesarik',2 \\ 'MARGen Clinic, Granada, Spain \\ ${ }^{2}$ Department of Biochemistry and Molecular Biology, University \\ of Granada, Spain
}

Correspondence: Jan Tesarik, MD, PhD, MARGen Clinic, Camino de Ronda 2, 18006 Granada, Spain, Tel +34 606376992 , Email jtisarik@clinicamargen.com

Received: October 21, 2018 | Published: November 16, 2018

Keywords: melatonin, cancer risk prevention, hormonal replacement therapy, menopausal hormone therapy, breast cancer, endometrial cancer

Abbreviations: MHT, menopausal hormone therapy; WHI, women's health initiative; ET, estrogen-only therapy; EPT, estrogen plus progestogen therapy; EMPT, estrogen-melatonin-progestogen therapy

\section{Introduction}

Based on several studies, including meta-analyses, published in the 1980s and suggesting that menopausal hormone therapy (MHT) could be beneficial for preventing osteoporosis, coronary heart disease and dementia, thus leading to decreased mortality, it was recommended by the American College of Physicians to be used to reduce the risk of coronary disease and other life-threatening conditions associated with menopause. ${ }^{1}$ However, since the designation, in 1998, of the Women's Health Initiative (WHI) trials to determine the benefits and risks of hormone therapy when taken for chronic disease prevention by predominantly healthy postmenopausal women, both the indications and contraindications of MHT, as well as the formulas of the hormonal preparations used, were undergoing substantial changes (reviewed in Manson et al. $)^{2}$. Several randomized trials, backed by WHI and carried out in the early 2000s, suggested that the risks of MHT outweighed any benefits. ${ }^{3,4}$ This led to a progressive decrease in the initial enthusiasm about the use of MHT, reflected by a $50 \%$ drop of its use in the period between 2002 and 2012. ${ }^{5}$

One of the major concerns about the use of MHT is the potential for an increased risk of breast cancer. ${ }^{6}$ This paper suggests that the recent discovery of the potential of melatonin to be used as a specific and nontoxic agent for causal therapy of different types of cancer, ${ }^{7}$ and breast cancer in particular, ${ }^{8}$ may invert this trend.

\section{Weighing up the pros and cons of MHT}

There are arguments both for and against the prescription of MHT There are two basic types of MHT: estrogen-only therapy (ET) and estrogen plus progestogen therapy EPT. ${ }^{9}$ Estrogen is the hormone that provides the most menopausal symptom relief, but may increase the risk of uterine (endometrial) cancer if administered alone, an effect that can be counteracted by progestogen. Hence ET was recommended for women without a uterus due to a hysterectomy, while EPT was preferred in all other cases. ${ }^{9}$ However, the addition of progestogen to estrogen in the EPT regimens, apart from protecting the endometrium against the carcinogenetic effects of unopposed estrogen, also appears to increase the risk of breast cancer as compared with ET regimens. Most of research into the development of the optimal MHT strategies in different clinical scenarios, carried out over the last 10 years, aimed at the definition of the best fitted formulas, doses and modes of application of estrogens and progestogens to reduce the risk of both types of cancer.

\section{Melatonin}

Several recent studies have shown that melatonin suppresses pathological deviations of cell signaling pathways involved in carcinogenesis and other invasive processes in the human body. These deviations alter the responses of the affected cells to external and internal stimuli so as to increase their freedom of movement, invasiveness and heterotopic proliferation. This applies both to different types of cancer, ${ }^{7,10-15}$ including breast cancer. ${ }^{8,16}$ and other invasive pathological processes unrelated to cancer, such as ademyosis or endometriosis. ${ }^{17,18}$ Melatonin can inhibit different cell signaling pathways involved in carcinogenesis, which can explain its relative versatility, underlying its action against different types of cancer. For example, melatonin inhibits proliferation and invasion of glioma cells via repression of miRNA-155, ${ }^{14}$ modulates tumor growth in PTEN-mutated gliomas, ${ }^{11}$ inhibits the proliferation of gastric cancer cells through regulating the miR-16-5p-Smad3 pathway ${ }^{13}$ and inhibits breast cancer cell invasion through modulating DF-1/KLF17/ID-1 signaling pathway. ${ }^{8}$ 
As compared with the large number of data about the effects of melatonin on breast cancer cells, relatively few is known about the effects of melatonin in endometrial cancer. Observational clinical studies suggest a significant correlation between melatonin deficiency and endometrial cancer, ${ }^{19,20}$ although the mechanism of this correlation is not clear. ${ }^{21}$ Several lines of evidence show that the pathophysiological mechanisms implied in breast cancer ${ }^{22}$ are similar to those acting in the malignant transformations of different other cell types $^{7}$ leading to the development of primary cancer in many other locations including the endometrium. ${ }^{23,24}$ Melatonin is known to inhibit most of these pathways in various cell types, ${ }^{7}$ although further studies are needed to evaluate its direct effects on endometrial cancer cells.

\section{The future}

The beneficial effects of MHT in menopausal women are well known. The main arguments against its wider use over extended periods of a woman's postmenopausal life are related to potential risks of certain types of malignant tumors, especially breast and endometrial cancer. According to the latest update (2018) of the American Institute for Cancer Research, breast cancer is the most frequent cancer in women worldwide ( $25.4 \%$ of all cancers), well ahead of endometrial cancer (5.3\%). Nonetheless, it was because of the supposedly increased risk of endometrial cancer that the original, simple ET formula of MHT was changed into EPT. However, the latter was subsequently shown to increase the risk of breast cancer, which was quite a lot more serious issue, leading to a global decline of MHT use. The current guidelines of the International Menopause Society recomment that if MHT is prescribed, it be commenced before the age of 60 , or within 10 years of menopause. ${ }^{25}$ The inclusion of melatonin, leading to the development and optimization of estradiolmelatonin-progestogen therapy (EMPT) formulas, might extend the use of MHT beyond these currently recommended limits. Due to its beneficial effects on a number of age-related diseases unrelated with menopause, ${ }^{26}$ EMPT can also be expected to improve the health status and the quality of life of menopausal women in general.

\section{Acknowledgments}

None.

\section{Conflict of interest}

The authors declare that they have no conflict of interest.

\section{References}

1. American College of Physicians. Guidelines for counseling postmenopausal women about preventive hormone therapy. Ann Intern Med. 1992;117(12):1038-1041.

2. Manson JE, Chlebowski RT, Stephanick ML, et al. Menopausal hormone therapy and health outcomes during the intervention and extended poststopping phases of the Women's Health Initiative randomized trials. JAMA. 2013;310(13):1353-1366.

3. Rossouw JE, Anderson GL, Prentice RL, et al. Risks and benefits of estrogen plus progestin in healthy postmenopausal women: principal results from the Women's Health Initiative randomized controlled trial. JAMA. 2002;288(3):321-333.

4. Beral V, Million Women Study Collaborators. Breast cancer and hormone-replacement therapy in the Million Women Study. Lancet. 2003;362(9382):419-427.

5. Lobo RA. Where are we 10 years after the Women's Health Initiative? $J$ Clin Endocrinol Metab. 2013;98(5):1771-1780.

6. Shairer C, Lubin J, Troisi R, et al. Menopausal estrogen and estrogen- progestin replacement therapy and breast cancer risk. JAMA 2000;283(4):485-491.

7. Bondy SC, Campbell A. Mechanisms underlying tumor suppressive properties of melatonin. Int J Mol Sci. 2018;19:2205.

8. El-Sokkary GH, Ismail IA, Saber SH. Melatonin inhibits breast cancer cell invasion through modulating DF-1/KLF17/ID-1 signaling pathway. $J$ Cell Biochem. 2018.

9. The North American Menopause Society. The 2017 hormone therapy position statement of the North American Menopause Society. Menopause. 2012;24(7):728-753.

10. Li Y, Li S, Zhou Y, et al. Melatonin in the prevention and treatment of cancer. Oncotarget. 2017;8(24):39896-39921.

11. Ma H, Wang Z, Hu L et al. The melatonin-MT1 receptor axis modulates tumor growth in PTEN-mutated gliomas. Biochem Biophys Res Commun. 2018;496(4):1322-1330.

12. Yang CY, Lin CK, Tsao CH, et al. Melatonin exerts anti-oral cancer effect via suppressing LSD1 in patient-derived tumor xenograft models. Oncotarget. 2017;8(20):33756-33769.

13. Zhu C, Huang Q, Zhu H. Melatonin inhibits the proliferation of gastric cancer cells through regulating the miR-16-5p-Smad3 pathway. DNA Cell Biol. 2018;37(3):244-252.

14. $\mathrm{Gu} \mathrm{J}, \mathrm{Lu} \mathrm{Z}, \mathrm{Ji} \mathrm{C}$. Melatonin inhibits proliferation and invasion via repression of miRNA-155 in glioma cells. Biochem Pharmacother. 2017;93:969-975.

15. Chovancova B, Hudecova S, Lencesova L, et al. Melatonin-induced changes in cytosolic calcium might be responsible for apoptosis induction in tumour cells. Cell Physiol Biochem. 2017;44(2):763-777.

16. Kubatka P, Zubor P, Busselberg D, et al. Melatonin and breast cancer: Evidences from preclinical and human studies. Crit Rev Oncol Hematol. 2018;122:133-143.

17. Qi S, Yan L, Liu Z, et al. Melatonin inhibits 17ß-estradiol-induced migration, invasion and epitelial-mesenchymal transition in normal and endometriotic endometrial epitelial cells. Reprod Biol Endocrinol. 16;(1):62.

18. Tesarik J, Mendoza-Tesarik R. Melatonin: the first noninvasive causal therapy for both endometriosis and adenomyosis? J Gynecol Women's Health, 2018;12:1

19. Grin W, Grünberger W. A significant correlation between melatonin deficiency and endometrial cancer. Gynecol Obstet Invest. 1998;45(1):6265 .

20. Viswanathan A, Hankinson SE, Schernhammer ES. Night shift work and the risk of endometrial cancer. Cancer Res. 2007;67(21):10618-10622.

21. Kobayashi Y, Itoh MT, Kondo H, et al. Melatonin binding sites in estrogen receptor-positive cells derived from human endometrial cancer. $J$ Pineal Res. 2003;35(2)71-74.

22. Bilyk O, Coatham M, Jewer M, et al. Epithelial-to-mesenchymal transition in the female reproductive tract: from normal functioning to disease pathology. Front Oncol. 2017;7:145.

23. Ma X, Ma CX, Wang J. Endometrial carcinogenesis and molecular signaling pathways. Amer J Mol Biol. 2014;4:3.

24. Liu Z, Qi S, Zhao X, et al. Metformin inhibits 17ß-estradiol-induced epitelial-to-mesenchymal transition via $\beta$ Klotho-related ERK1/2 signaling and AMPK $\alpha$ signaling in endometrial adenocarcinoma cells. Oncotarget. 2016;7(16):21315-21331.

25. Baber R, Panay N, Fenton A, et al. IMS Recommendations on women's midlife health and menopause hormone therapy. Climacteric. 2016;19(2):109-150.

26. Karasek M. Melatonin, human aging, and age-related diseases. Exp Geront. 2004;39(11-12):1723-1729. 